\title{
Differential Presentation of Congenital Cholesteatoma in Twins with Atypical Location
}

Udayabhanu H.N. ${ }^{1} \quad$ Lorenzo Lauda $^{1} \quad$ Antonio Caruso ${ }^{1} \quad$ Mario Sanna $^{1}$

Address for correspondence Udayabhanu H.N., MS, Gruppo Otologico C/o Casa di Cura, Via Emmanueli 42, Piacenza, Italy 29121 (e-mail: udayaentskullbase@gmail.com).

\begin{abstract}
Objective We present an extremely rare report of congenital cholesteatoma (CC) in identical twins with atypical location.

Methods Two consecutive cases of CC in identical twins from diagnosis to treatment and a literature review are presented.

Results The twin children presented to our otology and lateral skull base clinic with different clinical picture. Both underwent thorough audiological workup and imaging and early surgical intervention. The 6-year follow-up of these two children has been uneventful with no recurrence of disease and hearing restoration.

Keywords Conclusion Multiple theories exist to explain this rare pathology. Modified Leven-

- congenital son's criteria define it as white mass behind intact tympanic membrane. Atypical

- cholesteatoma

- twins

- middle ear location of CC with recurrent otitis media is not uncommon; hence, further study is required. To our best knowledge, this is the second reported occurrence in identical twins but first report with differential presentation and atypical location in both cases.
\end{abstract}

\section{Introduction}

Congenital cholesteatoma (CC) of the middle ear is a very rare pathology with no inflammatory process more frequently affecting boys. ${ }^{1}$ In identical twins, it can be described as rarest of rare occurrence. CC is an epidermoid cyst that develops behind an intact tympanic membrane (TM) in a patient with no history of otorrhea, trauma, or predisposing ear surgery. It is believed to occur due to straying remnant of embryonic epithelial tissue left after closure of the neural groove, which occurs between the third and fourth weeks of embryonic life. ${ }^{2}$ Derlacki and Clemis ${ }^{3}$ first defined this condition in 1965, with a modification introduced in 1986 by Levenson et al. ${ }^{4}$ The strict initial criteria were the presence of a white pearl behind an intact TM with no history of perforation, otorrhea, and otologic surgery. It is now accepted that cases with a history of acute otitis media, otitis media with effusion (OME), revealing otorrhea, or myringotomy should be included. ${ }^{2}$

In a study by Michaels 5 in the fetal temporal bones, he demonstrated an epidermoid structure between 10 and 33 weeks of gestation located in the anterosuperior part of the tympanum.
The persistence of this Michaels ${ }^{5}$ structure could act as an anlage and lead to CC in the anterior mesotympanum. CC in a child could be asymptomatic and diagnosed incidentally. It could present as hearing loss due to erosion of the ossicular chain or as recurrent attacks of OME due to occlusion of the tubal orifice by the cholesteatomatous mass. Asymptomatic CC could silently progress involving the mastoid space or causing TM perforation. ${ }^{2}$

Congenital cholesteatoma can lead to vertigo (if semicircular canals are eroded), fever, and headache due to intracranial complications. Facial palsy or sudden sensorineural hearing loss occur as result of progress of $\mathrm{CC}$ of the middle ear into petrous bone cholesteatoma (PBC). Otoscopy is characterized by pearly white mass bulging of the anterosuperior or posterior quadrant medial to an intact TM. ${ }^{2}$

The pediatrician or otologist should have high degree of suspicion to detect the presence of CC at early stage. In this article, we present $\mathrm{CC}$ occurring in identical twins in the same side ears (left)-an occurrence reported only once previously in the world literature. However, it is the first case report to our knowledge with atypical location involving the posterior mesotympanum.
DOI https://doi.org/ 10.1055/s-0038-1675663
Copyright @2018 Indian Society of Otology
License terms

() (1) $\Theta \circledast$ 


\section{Case Reports}

\section{Case 1}

A 7-year-old boy (first twin) attended our otology and lateral skull base outpatient clinic with history of one episode of otorrhea in the past as told by the informant (parent) in the left ear. On otoscopic examination, the right ear was normal whereas the left ear showed retraction of posterior quadrant of the TM. Incidental finding of tiny pearly white mass below the incudostapedial joint behind the intact but retracted TM was noted. In this quaternary referral center for otology, we had high suspicion of CC. Pediatric audiometry showed normal audiogram and tympanogram. Ipsilateral reflexes in the left ear were positive at 2,000 $\mathrm{Hz}(95 \mathrm{~dB})$ and 4,000 Hz (98 $\mathrm{dB}$ ) and absent at $500 \mathrm{~Hz}$ and $1,000 \mathrm{~Hz}$, whereas contralateral reflexes were positive at all the corresponding frequencies.

Three months later the hearing was normal, but the tympanogram showed negative pressure of $-185 \mathrm{~dB}$ Pascal with type B curve and absent ipsilateral and contralateral stapedial reflexes. High-resolution computed tomography (HRCT) scan of the temporal bones revealed a soft tissue mass in the posterior mesotympanum around oval window.

The child underwent exploratory tympanotomy and transcanal excision of the CC in total located inferior to the stapes crura. The ossicular status was normal, and chorda tympani nerve was preserved. Fascia and cartilage were used for reconstruction. Postoperative hearing was normal. The child has been on follow-up for 6 years with no residual or recurrence of the cholesteatoma with normal hearing status.

\section{Case 2}

The brother of the first twin, also 7-year-old boy (second twin), was brought to the otology clinic by the informant (parent) anxious as to whether this child could have similar problem as first twin. The second twin had no prior history of otorrhea or any ENT (ear-nose-throat) procedures in the past. On examination to our surprise, the left ear had retraction of the posterior quadrant of the drum with edema and otitis externa of the deep canal skin. Pediatric audiometry showed normal audiogram but with left B type impedance curve with absent stapedial reflexes. He was treated with topical antibiotics for 1 month, and at follow-up the audiogram was normal again. However, otoscopy showed a white mass in the posterior quadrant behind intact TM.

High-resolution computed tomography showed soft tissue opacification of the left middle ear around the incus and the stapes with involvement of left mastoid air cells. We advised mastoid exploration of the left ear. One month later when second twin came for surgery, the audiologic data were recorded and preoperative mean air-bone gap (ABG) was $47.5 \mathrm{~dB}$.

The second twin underwent canal wall up mastoidectomy with atticotomy. The incus was eroded and removed; stapes suprastruture was intact and normal. Cholesteatoma sac involving the mastoid and posterior mesotympanum was excised into to. Reconstruction was done with Silastic, fascia, and cartilage. Postoperatively, the mean ABG at 3 months was $55 \mathrm{~dB}$ with normal TM, and second-look surgery was advised for hearing restoration. One year later, the child underwent second-look surgery with type 2 ossiculoplasty and an epithelial pearl around stapes footplate was removed. The mean postoperative ABG after 5 years of follow-up is $42.5 \mathrm{~dB}$ with an improvement of $13.5 \mathrm{~dB}$ after second-look surgery. Both the twins have follow-up of 6 years with no recurrence of cholesteatoma (-Fig. 1).

\section{Discussion}

The prevalence of CC is as low as 2 to $5 \%$. CC may present at any age from infancy to adulthood as typical pearly white mass behind an intact TM. ${ }^{6}$ House and Sheehy ${ }^{7}$ have stated that the possibility of CC should be considered in patients with unilateral conductive hearing loss and without a clear diagnosis of OME, regardless of age. CC may be asymptomatic or alternatively may include conductive or sensorineural hearing loss, aural fullness, tinnitus, vertigo, labyrinthitis, facial palsy, and intracranial complications, depending on the location and extent of the lesion.

The pathogenesis of CC is not clear with multiple theories reported in the literature. Teed noted that failure of involution of an ectodermal epithelial thickening in proximity to the geniculate ganglion medial to the neck of malleus may constitute the CC source. ${ }^{8}$ Also, other etiological hypotheses include a tympanic ring defect, epidermoid formation, epithelial metaplasia, or implantation. ${ }^{9}$

The definition of CC by Derlacki and Clemis ${ }^{3}$ and later addition by Levenson et $\mathrm{al}^{4}$ has been challenged by McGill et $\mathrm{al}^{9}$, who have reported that approximately $70 \%$ children will have one episode of otitis media. In both our cases, contrary to few studies by Derlacki and Clemis ${ }^{3}$ (1965) and Levenson ${ }^{4}$ et al, CC were located in the posterior mesotympanum. As no existing theory can truly explain the origin of CC in the posterior location, a strong conjecture can be made that these lesions originate from embryonic epithelial cell rests that are trapped in the posterior mesotympanum during the development of the temporal bone, unlike Michael's ${ }^{5}$ structure that could explain CC in the anterosuperior mesotympanum. ${ }^{2}$

Zini and Sanna ${ }^{1}$ have classified CC into type A (mesotympanic), type $B$ (epitympanic), and type $A B$ (mixed). Type $A$ has been further divided into premalleolar (A1) and retromalleolar (A2). Both the cases in this study belonged to type A2 (retromalleolar). The dilemma faced by the otologist is to convince the parents of asymptomatic young children with CC that the surgical excision is the optimal therapy ${ }^{2}$ (-Fig. 2).

Owing to rapid tissue growth and Eustachian tube dysfunction, cholesteatomas in children have a more aggressive growth pattern relative to adults. Thus, incidence of both residual and recurrent diseases is higher in children compared with adults. ${ }^{2}$ Two main goals of surgery in children include eliminating disease completely to prevent recidivism and recurrence and preserving or restoring hearing. This has been achieved in these identical twins mentioned in our report by timely and appropriate surgical intervention with uneventful follow-up for 6 years. Early surgical intervention, which is the optimal therapy in CC avoids progression of disease and reduces the risk of complications and recurrence. 


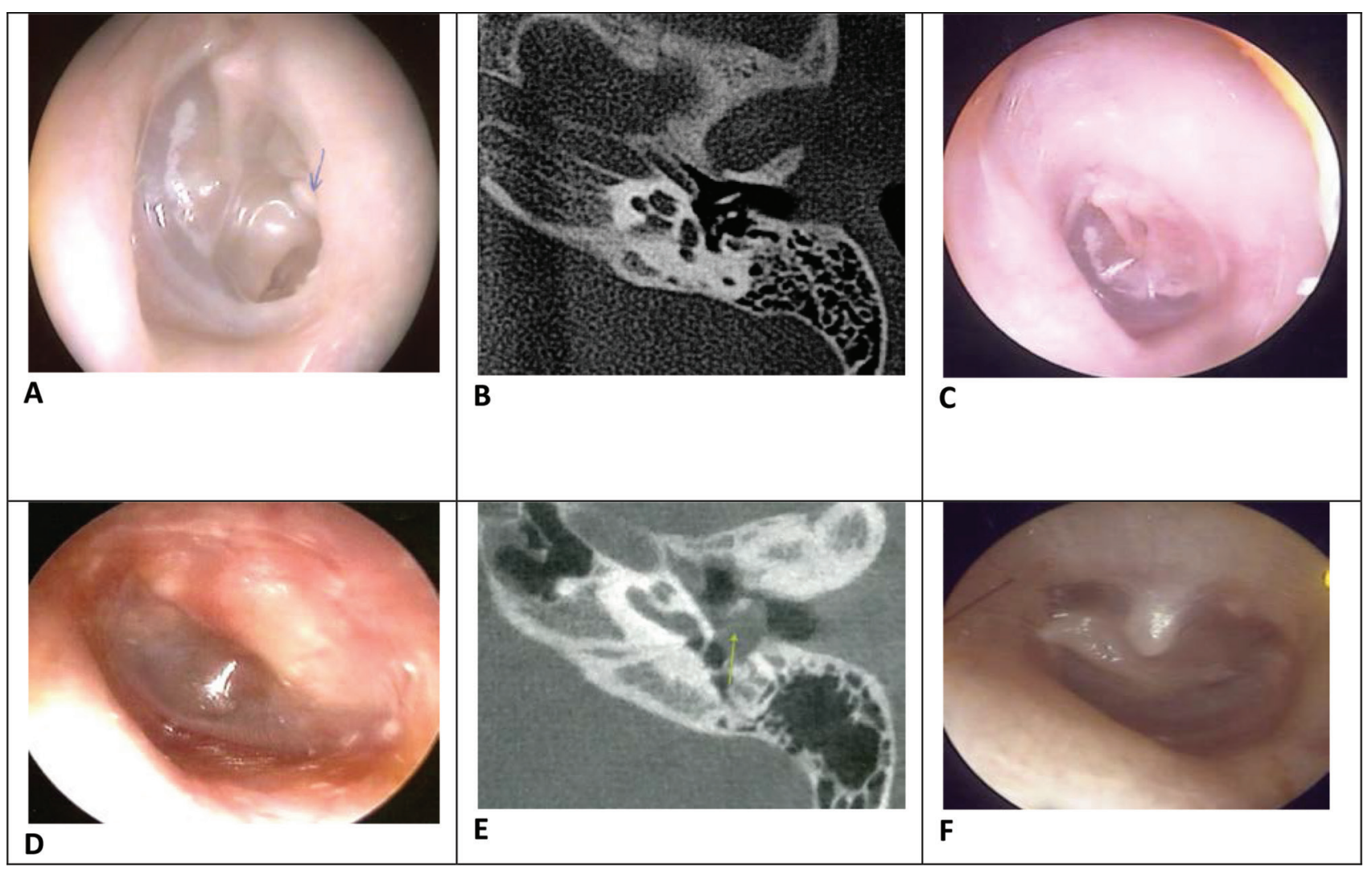

Fig. 1 Pre- and postoperative oto-endoscopic pictures with preoperative radiology of twins. (A) First twin's preoperative oto-endoscopic picture of left intact tympanic membrane with posterior quadrant retraction with CC. (B) First twin's HRCT of left temporal bone (axial image) showing CC in left posterior mesotympanum. (C) First twin's recent postoperative oto-endoscopic picture after transcanal excision of CC with 6-year follow up. (D) Second twin's preoperative oto-endoscopic picture of left intact tympanic membrane with posterior quadrant CC. (E) Second twin's HRCT of left temporal bone (axial image) showing CC mass in left posterior mesotympanum with incus erosion. (F) Second twin's recent postoperative oto-endoscopic picture after CWU tympanoplasty with type 2 ossiculoplasty (second stage) with 6-year follow-up.

\section{Conclusion}

Congenital cholesteatoma is a rare but aggressive disease, which may be asymptomatic in presentation. High degree of suspicion is required by the otologist to diagnose CC, and atypical location of $\mathrm{CC}$ in the posterior mesotympanum is quite common. CC in identical twins can be described as the rarest of rare occurrence. Early surgical intervention avoids progression of CC to involve the petrous bone and prevents complications. The goal of surgery is to avoid recurrence and restore hearing in a child.

\section{Summary}

- Congenital cholesteatoma in identical twins is the rarest of rare occurrence.

- In our report, we describe CC in identical twins with atypical location involving the posterior mesotympanum.

- Clinical presentation of CC can vary from being asymptomatic to include symptoms and signs such as hearing loss, tinnitus, vertigo, perforation of TM, and facial palsy, depending on the progression of the disease.

- High degree of suspicion with thorough aural examination is required to diagnose CC in children.

- The pathogenesis of CC is not clear with multiple theories.
- Early surgical intervention in CC is necessary to avoid complications.

- Excision of cholesteatoma sac in total is required to avoid residual and recurrent disease.

- Hearing preservation or restoration is one of the goals of surgery.

- Congenital cholesteatoma of the middle ear can be aggressive in nature progressing to involve the petrous bone.

\section{Conflict of Interest and Financial Disclosure}

This research received no specific grant from any funding agency, commercial or not-for-profit sectors. All financial and material support is by Gruppo Otologico, Casa Di Cura Hospital, Piacenza, Italy.

\section{Ethical Standards}

The authors assert that all procedures contributing to this work comply with the ethical standards of the relevant national and institutional guidelines on human experimentation and in accordance with institutional ethics committee of Gruppo Otologico, Casa Di Cura Hospital, Piacenza, Italy, and with the Helsinki Declaration of 1975, as revised in 2008.

\section{Conflict of Interest}

None. 


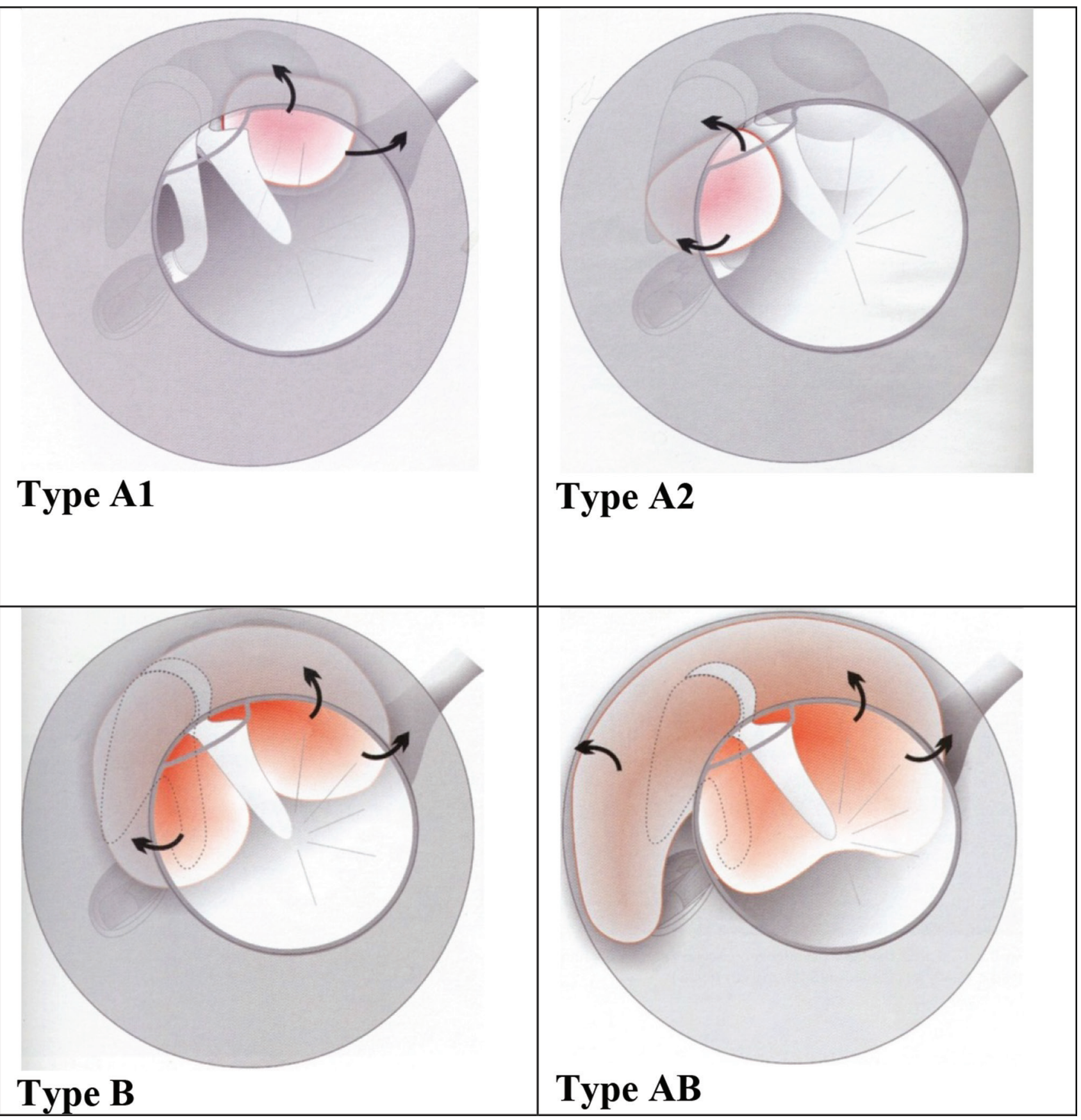

Fig. 2 Pictorial representation of Zini-Sanna classification of congenital cholesteatoma. A1, premalleolar; A2, retromalleolar; $\mathbf{B}$, epitympanic type; $\mathbf{A B}$, mixed type of congenital cholesteatoma.

\section{Acknowledgment}

I wish to sincerely thank the Department of Otology, Neurotology, and Lateral Skull Base surgery Gruppo Otologico, Casa Di Cura Hospital, Piacenza, Italy, for providing the support and the material for this study.

\section{References}

1 Sanna M, Zini C. "Congenital cholesteatoma" of the middle ear. A report of 11 cases. Am J Otol 1984;5(5):368-373

2 Sanna M, Caruso A, Russo A. Color Atlas of Endo-Otoscopy: Examination-Diagnosis-Treatment. 1st ed. New York, NY: Thieme publishers; 2017
3 Derlacki EL, Clemis JD. Congenital cholesteatoma of the middle ear and mastoid. Ann Otol Rhinol Laryngol 1965;74 (3):706-727

4 Levenson MJ, Parisier SC, Chute P, Wenig S, Juarbe C. A review of twenty congenital cholesteatomas of the middle ear in children. Otolaryngol Head Neck Surg 1986;94 (5):560-567

5 Michaels L. An epidermoid formation in the developing middle ear: possible source of cholesteatoma. J Otolaryngol 1986;15(3):169-174

6 Kojima H, Miyazaki H, Tanaka Y, Shiwa M, Honda Y, Moriyama H. [Congenital middle ear cholesteatoma: experience in 48 cases] [in Japanese]. Nippon Jibiinkoka Gakkai Kaiho 2003;106(9):856-865 
7 House JW, Sheehy JL. Cholesteatoma with intact tympanic membrane: a report of 41 cases. Laryngoscope 1980;90(1):70-76

8 Teed RW. Cholesteatoma Verum tympani: its relationship to the first epibranchial placode. Arch Otolaryngol 1936;24(4):455-474
9 McGill TJ, Merchant S, Healy GB, Friedman EM. Congenital cholesteatoma of the middle ear in children: a clinical and histopathological report. Laryngoscope 1991;101(6 Pt 1):606-613 